\title{
A Cognitive Routing Framework for Reliable Communication in IoT for Industry 5.0
}

\author{
Saptarshi Ghosh, Student Member, IEEE, Tasos Dagiuklas, Senior Member, IEEE, Muddesar Iqbal, Member, IEEE \\ Xinheng Wang, Senior Member, IEEE \\ \{ghoshs4, tdagiuklas, m.iqbal\}@1sbu.ac.uk ,xinheng.wang@xjtlu.edu.cn
}

\begin{abstract}
Industry 5.0 requires intelligent self-organized, selfmanaged and self-monitoring applications with ability to analyze and predict both the human as well as machine behaviors across interconnected devices. Tackling dynamic network behavior is a unique challenge for IoT applications in industry 5.0. KnowledgeDefined Networks (KDN) bridges this gap by extending SDN architecture with Knowledge Plane (KP) which learns the network dynamics to avoid sub-optimal decisions. Cognitive Routing leverages the Sixth-Generation (6G) Self-Organised-Networks with self-learning feature.

This paper presents a self-organized cognitive routing framework for a KDN which uses link-reliability as a routing metric. It reduces end-to-end latency by choosing the most-reliable path with minimal probability of route-flapping. The proposed framework pre-calculates all possible paths between every pair of nodes and ensures self-healing with a constant-time convergence. An experimental test-bed has been developed to benchmark the proposed framework against the industry stranded Linkstate and distance-vector routing algorithms SPF and DUAL respectively.
\end{abstract}

Index Terms-Industry-5.0, Cognitive Routing, Rapid Convergence

\section{INTRODUCTION}

$\mathbf{I}$ N 2013, the German Academy of Engineering Sciences presented a recommendation and research agenda for Industry 4.0. Its primary motivation was to achieve seamless integration between physical and virtual technologies to facilitate smart manufacturing, which results in significant inflation of the IoT technology in industrial automation. Between 2009 and 2019, the Industrial sector has contributed $20 \%$ to the EU's GDP. Industry 5.0, as a natural successor, aims to build on top of the existing architectural frameworks of Industrial and Heterogenus IoT (I-IoT, H-IoT) and interoperability between cyber-physical systems. The Directorate-General of Research and Innovation (EU) has identified a new set of concepts that Industry 5.0 addresses. These are Human-centric solutions, Bio-inspired Technologies, Real-time digital-twins technology, Network analytics, Machine-learning based automation, and Trustworthy autonomy. A large-scale industry needs to have a scalable network fabric to interconnect all its devices. Software-Defined Networking (SDN) provides such a programmable, vendor-agnostic communication platform. 5G leverages SDN at its core to virtualize network services (NFV),

S. Ghosh, T. Dagiuklas and M. Iqbal with the School of Engineering, Division of Computer Science and Informatics, London South Bank University, London, UK

X. Wang is with the department of Mechatronics and Robotics, School of Advanced Technology, Xi'an Jiaotong-Liverpool University and ISPs use it in WAN deployment (SD-WAN). SDN provides a bird's eye view of the network where the control plane accumulates global knowledge about the underlying topology and flows. Additionally, the data plane generates enough which the controller can mine for analytics. In SDN-based routing, the routing protocol uses the global view to calculate optimal paths without letting the routers exchanging control packets. An efficient routing protocol aims to avoid sub-optimal paths and converge rapidly in a dynamic environment. However, highly time-critical industrial communication systems, such as IoT infrastructure for manufacturing plants, can not tolerate delays due to routing protocol convergence. Therefore, routing optimization using analyzing the network's behavior provides a better heuristic which eventually reduces the convergence probability.

In SDN [1] Routing, the Shortest-Path calculation is the subjected Optimization problem where a controller calculates the optimal values of the free parameters subject to a set of communication constraints defined as a policy (SelfOptimization). The controller then Configures the parameters into the underlying network devices (Self-Configuration) and serves alternate Routes On-Demand, if the primary one fails (Self-Healing); thus supporting the SON [2]. However, the application of Machine Learning (ML) in Route-Optimization is a relatively new domain; at the time of writing this paper, there are a handful of works done in developing an Intelligent Routing Algorithm for SDN. The base model of fitting ML in $\mathrm{SDN}$ is referred to as Knowledge-Defined Networks (KDN) [3], where the primary objective is to accumulate holistic information from a supervising Control Plane (CP) of an underlying IP-network, analyze them to extract knowledge that generalizes the network behavior. This knowledge eventually helps to bypass the need for using costly heuristic Routing algorithms, having preserved the equal adaptation capabilities to network dynamics [4].

Self Organized Networking (SON) [5] in the fifth-generation cellular communication systems $(5 \mathrm{G})$ enhances the requirements of its predecessor. Some of the new requirements involve increasing traffic capacity, improving QoS/QoE, support of heterogeneous Radio Access Networks (RAN), 10Gbps peack data rate, sub-millisecond latency, support of ultrahigh reliability, improved security, privacy and flexibility, and reduction of CAPEX and OPEX [6] [7] [8]. SON constitutes the following three entities.

- Self-Optimization provides several control-plane (CP) optimization strategies such as Caching, Routing, load- 
balancing, etc. which are invoked autonomously. Relevant algorithms calculates the optimal values of several decision variables w.r.t. the a set of constrains, called policies.

- Self-Configuration automates the injection of the decision parameters (e.g. operational and radio config) to the underlying data-plane devices.

- Self-Healing provides high-availability to the overall network. A typical model uses detection, diagnostic and compensation sequence to automate the recovery process.

Recent development in SON shows a significant use of ML to accelerate the performance of its constituents [9].

In this paper, we propose Most-Reliable-Route-First (MRRF), an Intelligent Routing algorithm for Self-Organised Knowledge-Defined Networks (SO-KDN). The proposed model initially calculates all possible paths for all pairs of nodes from the Networks' topology using the proposed algorithm (MRoute) and aims to learn the reliability of individual links by their statistical measures of volatility over time. The algorithm maintains the rank of the routes based on their cumulative reliability and serves them on-demand in constant time, hence assuring the most reliable Routes. We further propose a full-fledged implementation of the KDN model as a test-bed to conduct experiments, which benchmarks MRoutes with Diffusion Update Algorithm (DUAL) [10] and Shortest Path First (SPF) [11] that powers EIGRP as OSPF respectively. The rest of the paper is organised as follows.

Section II introduces elementary concepts of 6G, Section III presents the state of the art of intelligent SDN-Routing, The system model including problem formulation, the design $\&$ analysis of the proposed algorithm is discussed in Section IV, Section V addresses the ML extension and the learning process, The experimental setup, Hyper-parameter tuning, benchmarking and the analysis of results have been provided in section VI and finally, we conclude the article in section VII.

\section{STATE OF THE ART IN KDN}

The inception of the KDN comes from the work of Clark et. al. [3], where he proposes a unified Knowledge Plane (KP) that takes decisions based on partial and conflicting information, accumulated from a distributed cognitive framework. The paper considers the use of KP in solving the Optimal RoutePreference problem by learning network behavior over time. However, the paper lacks information to real-world network types such as ISP, Enterprise, Cellular, etc., and does not include working principles in a heterogeneous networks. These issues are addressed by Strassner et. al. [12] by their extension of KDN with an Interface-Plane, that offers a much clear view of the implementation and necessary building blocks. Several surveys show the growing application of ML and Deep Learning (DL) on SDN architectures in recent times, that aims to achieve the KDN. Fadlullah et. al. presents a classification of various ML/DL algorithms and their application to intelligent network traffic control systems [13]. Chen et. al. focuses on the application of DL into several Cognitive Wireless communication systems such as the Internet of Things (IoT), Mobile Edge Computing (MEC), Unmanned Areal Vehicle (UAV) networks, etc [14]. Zhao et. al. [15] reviews the specific applications of ML into SDN problems such as defense mechanism from Distributed Denial of Service (DDoS) attacks [16], Anomaly Detection, Traffic Classification, Routing Optimization, etc. To restrict our scope of the discussion, we now put the relevant state of the art focusing on Routing Optimization only.

Shortest Path Algorithm (SPA) and Heuristic Algorithms (HA) are the two widely used approaches that solve RoutingOptimization problems [17]. Among several alternatives, Artificial Neural Networks (ANN), Reinforcement Learning (RL), Deep RL (DRL), and Lazy Learning (LL) are the four learning models primarily used to address Routing Optimization. Yanjun et. al. [18] proposes an ML-Meta later based approach where an ML model is trained by the calculated traffic parameters of a heuristic algorithm and its corresponding network state as input. The proposed framework maps the input and output of the HA that reduces its exponential run-time in a constant one. NeoRoute [19] models traffic characteristics by forecasting future link consumption using the Recurrent Neural Network (RNN) with Long Short-Term Memory (LSTM). A similar problem is addressed by Álvaro López-Raventós, et. al. for high-density WANs [20]. The aforementioned works use Supervised-ML models for training, which assumes the network characteristics are likely to stay identical over time. Therefore, they are not suitable for dynamic networks, which in contrast needs an Online-Training model such as RL or DRL. Sandra et.al. [21] propose a DRL framework, trains an agent that weight the delay, loss, and bandwidth for every possible link of a target network. The network feeds either reward or penalty back to the agent based on the change in end-to-end throughput. The agent uses the feedback to tune its decision-making model. Francois [22] et.al. applies DRL with a Random Neural Network in cognitive-routing in SDN. The proposed architecture shows consistent performance even in a highly chaotic environment. Applications of DRL in SDN specific problems include QoS Aware Adaptive Routing [23].

In this research we use a Time-Series analysis model that extracts link volatility trends using RNN+LSTM to ensure reliability in constant time.

\section{SySTEM MODEL}

Programmable networks consist of a topology of configurable routers. Routers connect the a Local Area networks (LAN) to interface with the switching networks and Wide Area Networks (WAN) to interconnect with neighbouring routers. In a heterogeneous routing environment, the routers' computational capacity varies significantly. The load on a route processor delays the control-packets processing (service delay or node-cost) as most of the data-plane traffic are switched at the router Application Specific Integrated Circuits (ASIC). However, existing enterprise routing protocols (e.g., EIGRP, OSPF) don't include service delay as a metric parameter. That said, the link-cost i.e., the propagation and transmission delay is influenced by several link parameters such as throughput, latency, load and reliability. QoS aware routing protocols uses admission control mechanism to allow only the traffics that meets certain constraints specified in the policies. In the 
proposed system model, routers have a node-cost and the linkcosts are calculated by optimizing the respective objective function subject to a set of link-specific constraints. The proposed routing model uses both node and link costs as metric parameter. This offers a novel routing model which includes service delays in route calculation also complying with the QoS routing principle. The routing algorithm (MRoute) proactively enumerates all-possible paths between all-pairs of nodes. Further, it the varying costs is used to calculate link-specific reliability. The model uses the reliability as a metric for routing and provides routes on demand. As the paths are proactively calculated, thus, the convergence does not need re-computation of the topology, hence results in constant time convergence.

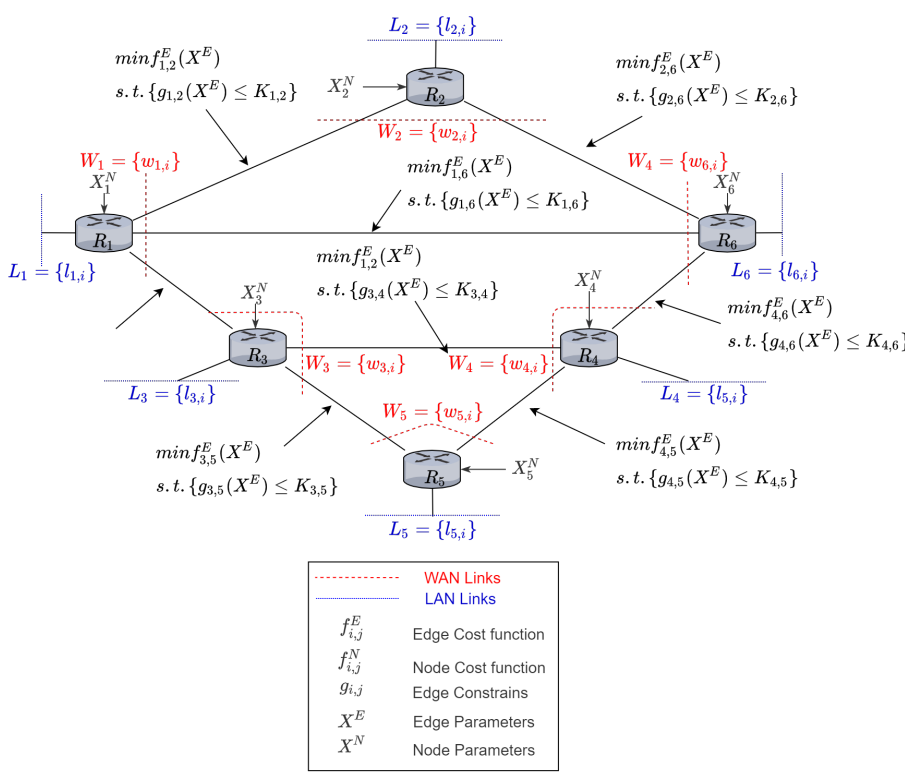

Fig. 1. Reference topology with route-policies

We choose to model the topology of a Software-Defined Network as a Simple, Finite, Connected graph. The network consists of programmable Routers and Switches, which are connected to the Controller via a secure and reliable SouthBound Interface (SBI). The controller treats both the Router and Switch as a generic Edge-Node (EN) having a welldefined set of Communication (L1) and MAC (L2) protocols configured. Additionally, the Routing (L3) and Transport (L4) protocols must ensure the following properties. Edge-Nodes don't exchange Control-Plane traffic among each-other but only with the controller over the SBI. There exists no Neighbour Discovery mechanism, Edge-Nodes share their local information and keep-alive packets with the controller only. Controller accumulates various telemetry information of the Edge-Nodes such as Memory, CPU, Network interface, etc. The network topology does not change frequently.

Each edge node $E N_{i}$ maintains a local routing table $\left(R T_{i}\right)$ that comprises of three disjoint sets of entries, The Connected Routes $\left(C R_{i}\right)$ which are networks, connected directly to the device interfaces, The Static Routes $\left(S R_{i}\right)$ are configured statically on the device and Remote Routes $\left(R R_{i}\right)$ are not learnt from the controller. By definition, these sets partition the routing table, i.e. $C R_{i} \cap S R_{i} \cap R R_{i}=\phi$ and $C R_{i} \cup S R_{i} \cup R R_{i}=$ $R T_{i}$. The controller uniquely identifies each edge-node by theirNodeID similar to Router ID in OSPF and EIGRP and maps it with their corresponding $C R$ set. When an edgenode receives a packet with unknown destination address, it forwards it to the controller. The controller then resolves the destination node's ID from from map, finds a route between the source and destination router and replies it back to the source node [24].

Figure 1 depicts a reference topology of 6 routers with Node ID $R_{1}-R_{6}$, the corresponding $C R_{i}$ s are further segregated into the LAN $\left(L_{i}\right)$ and WAN $\left(W_{i}\right)$ links $\left(L_{i} \cap W_{i}=\phi\right)$ following RFC-1918[]. The controller uses Link-State Routing (LSR) approach to build a topology from these information, i.e. nodes having common WAN network are adjacent. However, for the sake of simplicity, we did not include topology with Broadcast segments as it requires additional Designated node's placement [25]. Hence, we assume all the links are Point-to-Point in nature.

The network has a set of node-specific parameters $\left(X^{N}\right)$ such as, CPU and memory utilization, and a set of Edgespecific parameters $\left(X^{E}\right)$ such as, bandwidth, delay, load, reliability etc. The WAN links are constrained and heterogeneous i.e. its attributes are bounded above by some pre-defined values specific to that link. These values are generally dependent on the network policy or the media type, hence we leave it to be as user-defined. We propose the formulation of link-cost as a set linear pogramming Problems, for individual edges, with a linear cost-function $f_{i, j}^{E}: X^{E} \rightarrow \mathbb{R}^{+}$, between $R_{i}$ and $R_{j}$, such that its linear constrains $g_{i, j}\left(X^{E}\right) \leq K_{i, j}$ are met. This is to overcome the limitation of OSPF's sub-optimal routing issue due to its simplistic metric, and EIGRP's routeflapping problem caused by its dynamic metric parameters. The proposed method uses link attributes defined by RFC7868 [26]. However, as the metrics are calculated locally to the controller, it diminishes any need of exchanging updatepackets between edge-nodes, thus eliminates the cause of route-flapping. Similar to the edge-cost, the node-cost also contributes to the calculation of the final metric. The nodecost function $f_{i}^{N}: X^{N} \rightarrow \mathbb{R}^{+}$computes a cost based on the node attributes $\left(X^{N}\right)$. In our previous work [27] we have shown the benefits of routing optimization by fusing Node and Edge costs in metric calculation.

The controller generates a Graph structure isomorphic to the network topology, and weighs its edges by relaxing the $f_{i}^{N}$ and $f_{j}^{N}$ into $f_{i, j}^{E}$ for all adjacent $R_{i}, R_{j}$ Stochastic Temporal Edge Normalization (STEN) [27]. As the $X^{E}$ and $X^{N}$ varies over time but the topology remains same, hence the subjected Graph be a dynamic isomorphic Graph, which we refer to as Meta-Graph.

The proposed algorithm performs the following steps in order to meet the rapid-convergence criteria. First, it efficiently computes all possible paths between all-pairs of nodes from the meta-graph using an algorithm called MRoute. This step is invoked whenever the topology changes. Seconds, it Computes the reliability of the links by profiling their cost variation over the time using an RNN using LSTM; This is a periodic step. 
Third, it Ranks the computed paths obtained from step 1 based on their cumulative reliability obtained from step 2 . This step is invoked every time an update happens. First, Returns the most reliable routes on demand as primary route keeping the rest in backup. In case the primary route fails, next best route is served instantly. Hence the rapid-convergence is achieved.

The following sub-sections explain the problem formulation in details.

\section{A. Problem Formulation}

The Simple, Undirected and Connected Graph $G(V, E)$ represents the topology of the underlying network, where $V=\left\{v_{i}\right\}$ and $E=\left\{e_{i, j} \mid \operatorname{adj}\left(v_{i}, v_{j}\right)\right\}$ are the Vertex and Edge set respectively. $V$ and $E$ are finite and non-empty, $\operatorname{adj}\left(v_{i}, v_{j}\right)=1$ if $v_{1}, v_{j}$ are adjacent, and 0 otherwise. The graph is simple (No self-loop, no parallel edge) as to fit in the Shortest-Path Algorithm (SPA) criteria. It is undirected as we assume that the links are full-duplex in nature and the connected property ensures that a path between any pair of vertices. The following measures are computed from $G$ :

1) Adjacency Matrix: : $\operatorname{ADJ}(G)=[\operatorname{adj}(i, j) \in\{0,1\}]^{n \times n}$ is a symmetric binary matrix represents the adjacency of the $G(V, E)$, where $|V|=n$.

2) Policy Set: : Is a finite, non-empty set of policy tuples that includes $f_{i, j}^{E}$ and $\left\{g_{i, j} \leq K_{i, j}\right\}$ (Equation 1)

$$
P L C=\left\{\left\langle f_{i, j}^{E}\left(X^{E}\right),\left\{g_{i, j}\left(X^{E}\right) \leq K_{i, j}\right\}\right\rangle \forall(i, j) \in E\right\}
$$

3) Variable Cost Matrix: $\operatorname{VCOST}(t)=\left[c_{i, j}(t) \in \mathbb{R}^{+}\right]^{n \times n}$ represents the cost matrix at time instance $t$ (Eq. 2),

$$
\left\{c_{i, j}(t)\right\}=\left\{\begin{array}{ll}
\min f_{i, j}^{E}\left(X^{E}, t\right) & \text { if } i \neq j,(i, j) \in E \\
f_{i}^{N}\left(X^{N}, t\right) & \text { otherwise }
\end{array}\right\}
$$

All the $n$ diagonal values $c_{i, i}$ represents corresponding node-costs $f_{i}^{N}$ and the non-diagonal ones represent the edge-costs $f_{i, j}^{E}$ for all valid edges i.e. $(i, j) \in E$.

4) Normalized Cost Matrix: As the diagonal elements of $V \operatorname{COST}(t)$ represents weighted self-loops, it violates the "simple-graph" criteria. Therefore, a normalization is needed that relaxes the self loops but preserves their effects onto the resultant "Simple-Graph". We use Stochastic Temporal Edge Normalization (STEN) [27] technique to do so, which results $\operatorname{NCOST}(t)=\left[\left\{c_{i, j}^{\prime}(t) \in[0,1]\right]^{n \times n}\right.$

5) Route Tree: The RouteTree $T_{s, d}$ is an m-way search tree that represents all possible paths between $v_{s}, v_{d} \in V$, it holds the following properties. The destination vertex $v_{d}$ is placed at root, all the leaves are identical i.e. the source vertex $v_{s}$, every unit-branch $\left(v_{i}, v_{j}\right)$ is weighed by its corresponding values in

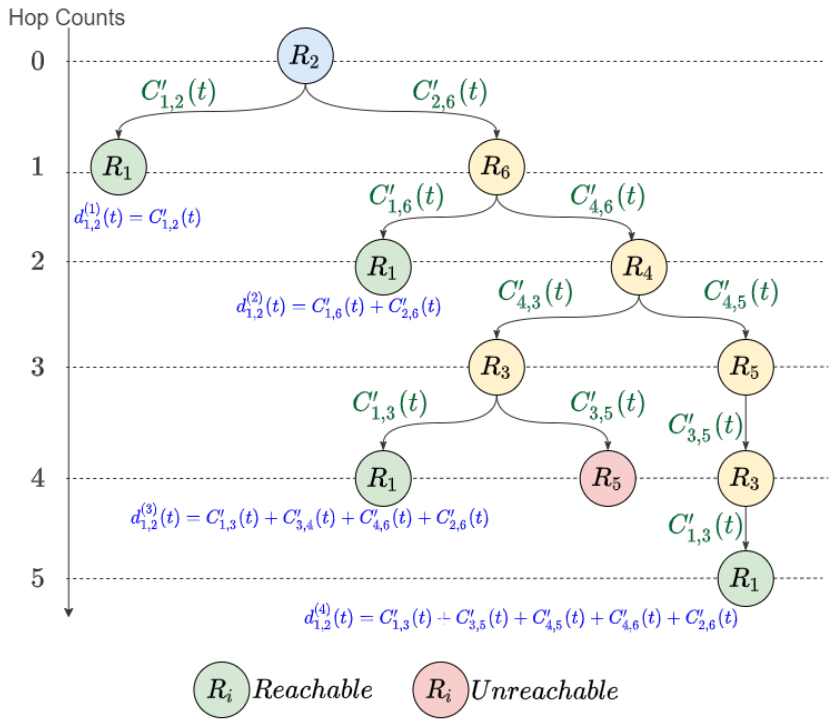

Fig. 2. RouteTree of $T_{1,2}$, rooted at $R_{2}$ all the reachable paths terminates with $R_{1}$ and unreachable node $R_{5}$

$N C O S T[i, j]$ and varies over time, and for any intermediate vertex $v_{k}$, if $A N S C\left(V_{k}\right)$ and $\operatorname{DESC}\left(V_{k}\right)$ denotes its ancestors and descendants, then $\operatorname{ANSC}\left(V_{k}\right) \cap \operatorname{DESC}\left(V_{k}\right)=\phi$, this prevents any loop.

The MRoute algorithm generates the tree and is discussed in the section III.C. Figure 2 depicts the RouteTree $T_{1,2}$ w.r.t. the reference topology Figure 1, it shows the hop-counts and cumulative costs for each valid route (terminating at source vertex $\left.v_{1}\right)$. At hop-count $=5, R_{3}$ has two children, $R_{1}$ and $R_{5}$, as $R_{1}$ is source, it terminates the search successfully. however, $R_{5}$ has no adjacency left that has not appeared in its ancestor set, therefore $\operatorname{adj}\left(R_{5}\right)-\operatorname{ANSC}\left(R_{5}\right)=\phi$ and the search registers an unsuccessful termination. The MRoute algorithm has two phases: Phase-1 (Grow Phase) where the tree grows recursively, where it registers several unsuccessful terminations, the Phase-2 (Shrink Phase) eliminates all such branches.

6) Route Forest: : For an $n$-node graph, there exists $\left(\begin{array}{l}n \\ 2\end{array}\right)$ possible pairs of nodes. Each node produces a RouteTree. A collection of such trees form a RouteForest. It is generated by invoking MRoute parallelly $\left(\begin{array}{l}n \\ 2\end{array}\right)$ times for each pair of nodes. The concurrency in execution is possible as the procedures are computationally independent and only the shared datastructures are read.

\section{B. Metric Formulation}

We propose a composite metric for MRoute that constitutes of the node cost $C_{i}^{N}(t)$ and edge costs $C_{i, j}^{E}(t)$. The node and edge parameters are listed in Table-I. The following paragraph are the formulation of Node and Edge costs.

1) Node Cost: The node cost uses CPU and memory utilization as parameters. However, CPU \& memory utilization can't solely determine performance (i.e. a $20 \%$ utilized 8 -core CPU processes more operations than that of a $80 \%$ single-core CPU and the same applies to the context of DDR4 vs DDR2 


\begin{tabular}{|l|l|l|l|l|l|}
\hline \multirow{3}{*}{ Node Parameters $\left(X^{N}\right)$} & \multirow{2}{*}{ CPU } & Parameter & Core Count $(n c)$ & Frequency $(f c)$ & Utilization $(u c)$ \\
\cline { 2 - 6 } & \multirow{2}{*}{ Memory } & Unit & Integer & MHz & {$[0,1]$} \\
\cline { 2 - 7 } & & Unit & MB & Frequency $(f m)$ & Utilization $(u m)$ \\
\cline { 2 - 7 } & & PHz & Volume $(v m)$ & {$[0,1]$} \\
\hline \multirow{2}{*}{ Link Parameters $\left(X^{E}\right)$} & Parameter & Bandwidth & Delay & Load & Reliability \\
\cline { 2 - 6 } & Units & Mbps & ms & {$[0,1]$} & {$[0,1]$} \\
\hline
\end{tabular}

TABLE I

LINK AND NODE PARAMETERS, MONITORED BY CP

memory). Moreover, with recent adaptation to network virtualization (eg. Cisco IoU, CSRv), CPU and memory allocation is more flexible, it yields more heterogeneity in the network. Therefore, we propose a more robust metric formulation. The weight parameters $\alpha_{c}$ and $\alpha_{m}$ are left to the user to regulate (e.g. EIGRP K-Valeus), the default value is set to 0.5.

$$
\begin{gathered}
C_{i}^{N}(t)=f_{i}^{N}\left(X_{i}^{N}, t\right)=\left[\alpha_{c}\left(f c_{i}(t) n c_{i}(t) u c_{i}(t)\right)+\right. \\
\left.\alpha_{m}\left(f m_{i}(t) v m_{i}(t) u m_{(i)}\right)\right]
\end{gathered}
$$

2) Link Cost: The link cost function uses parameters same as of EIGRP's. All the control traffic is targeted to the controller. This not only reduces the diameter of control flow from $O(n)$ (linear) to $O(1)$ constant, but also results in fast convergence. It is due to the fact that the topology is built inside the controller's memory and no control-packets are flooded to build neighbourship. The SDN paradigm unifies the benefits of both OSPF and EIGRP as it builds a complete topology view like OSPF and uses all parameters of a more robust composite metric and supporting unequal-cost load balancing like EIGRP (Discussed in subsection E).

$$
\begin{aligned}
C_{i, j}^{E}(t) & =f_{i, j}^{E}\left(X_{i, j}^{E}, t\right)=\left[\left(\beta_{L} M L D_{i, j}(t)\right) \times\right. \\
& \left.\left(\beta_{B} B W_{i, j}(t) \beta_{D} D L Y_{i, j}(t)\right) \times \beta_{r}\left(1-R L Y_{i, j}(t)\right)\right]
\end{aligned}
$$

The formulation in Equation 4 is realised by its three components (BDP, Load and Reliability). The Bandwidth Delay Product $B D P=B W(t) \times D L Y(t)$, measures the instantaneous end-to-end link capacity. The BDP is scaled by the mean load (occupancy $=B D P(t) \times M L D(t))$ and measures the amount of the occupancy in the link.The occupied capacity is scaled with additive inverse of reliability (occupency $\times(1-R L Y)$ ) measuring the unreliability of the occupied capacity.

3) Normalized Metric: With reference to equation 3 and 4, the cumulative metric for a link $c_{i, j}^{\prime}(t)$, is obtained by relaxing the node costs of both endpoints $\left(C_{i}^{N}(t), C_{j}^{N}(t)\right)$ and scalling them by their corresponding load-share $\left(P_{i, j}(t), P_{j, i}(t)\right)$ into the link cost $C_{i, j}^{E}(t)$ (Equation 5). The parameter $\gamma_{N}, \gamma_{E}$ are weighing factors, set by the user. The load-share of an interface is a proportion of the number of packets passes through that interface over the total packet exchanged, The value is expressed in $[0,1]$.

$$
c_{i, j}^{\prime}(t)=\left[\gamma_{N}\left(P_{i, j} C_{i}^{N}(t)+P_{j, i} C_{j}^{N}(t)\right)+\gamma_{E}\left(C_{i, j}^{E}(t)\right)\right]
$$

Figure 3 depicts the relaxation process to calculate cumulative metric $c_{i, j}^{\prime}(t)$ at time $t$.

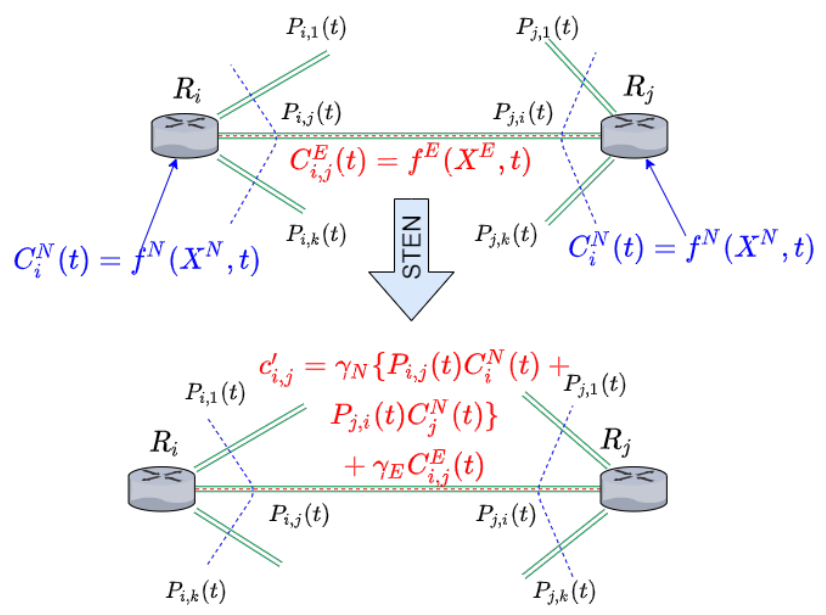

Fig. 3. Relaxation of Node costs into Edge using STEN [27] $T_{1,2}$

\section{Algorithm Design}

The proposed algorithm 1 is called MRoute, it takes source and destination vertex $\left(v_{s}, v_{d}\right)$ as input, looks up to global structures $A D J, N C O S T$ during its recursive run-time and returns a RouteTree $T_{s, d}$. The n-ary tree is stored into a hasheddynamic array structure. It finds all possible paths between a pair of vertices using Backtracking strategy. The problem is inherently brute-force in nature and the state-space complexity is NP-hard, therefore we introduce optimisation and relaxation which is further explained in the later section of this paper.

1) Optimising Data-structures: MRoute adds nodes recursively into the RouteTree, the algorithm assumes $\operatorname{ANS}\left(V_{k}\right)$ is of $O(1)$. Generally, an n-ary tree can be stored using either linked (non-contiguous) or array (contagious) structure. Since the data structure is unordered, each node must maintain $(|V|-1)$ pointers it consumes in $O\left(n^{2}\right)$ space. However, not every time the network is mesh. Additionally, the recurrence decreases monotonically as more neighbours are visited, they would not appear as children. Therefore, the number of children decreases as the tree gets deeper, and choosing a n-ary tree structure is not space-optimal.

In this article, we propose an optimal data structure to accommodate such a sparse array. Furthermore, when a graph is converted into tree, there will be multiple instances where the same node appears in various spaces. To eliminate any confusion during insertion, pointing and displaying a node, an 


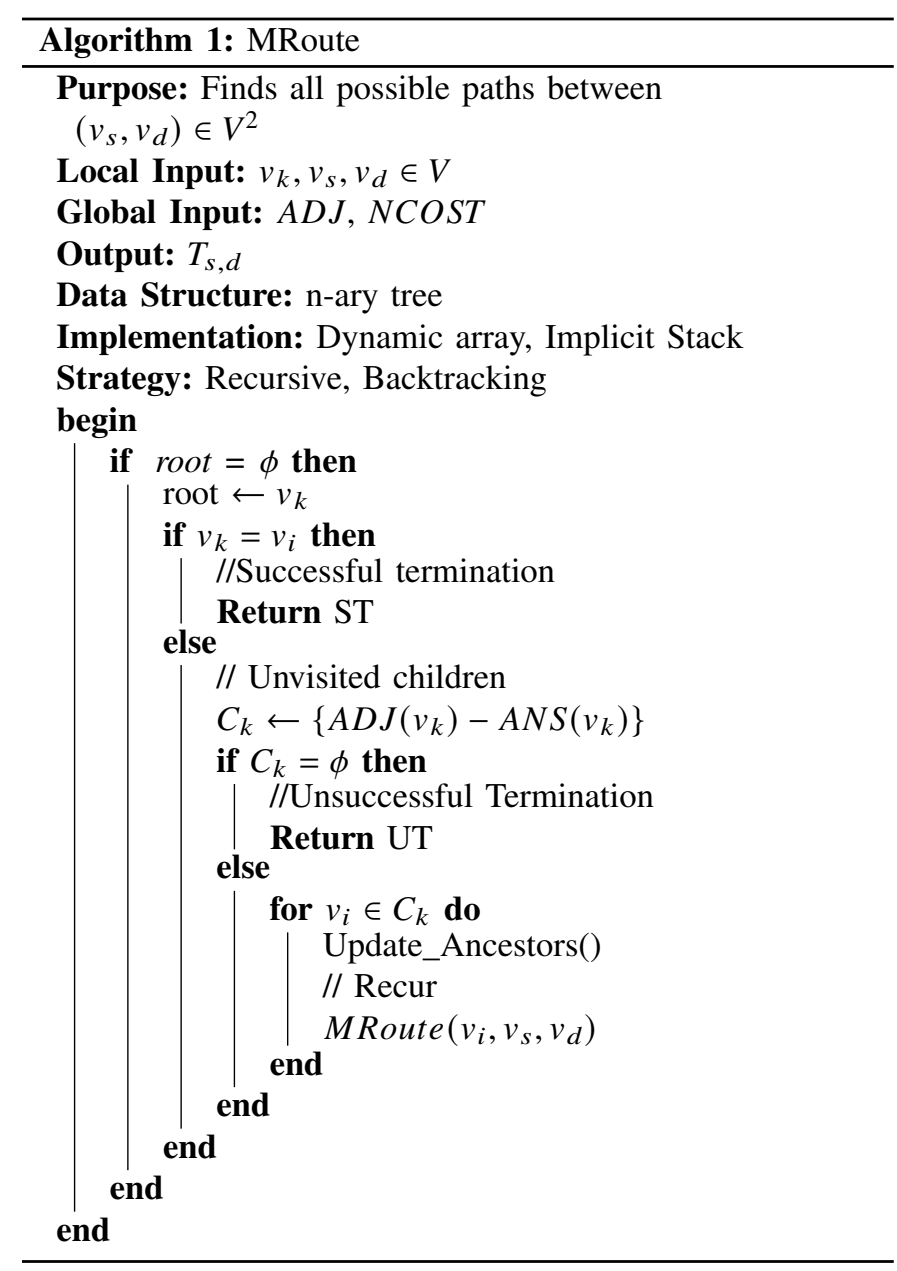

efficient and light index generation method is needed. For an nary tree the following (Equation 6) generalised heap-indexing rule is adapted for this purpose.

$$
\begin{aligned}
& \text { if } \operatorname{index}\left(v_{k}\right)=i \text { then } \operatorname{Parant}\left(v_{k}\right)=\left\lfloor\frac{i}{n}\right\rfloor, \text { and } \\
& \operatorname{Child}\left(C_{k, j}\right)=n i+j \operatorname{jindex}(\text { root })=0, n=|V|
\end{aligned}
$$

To avoid any segmentation error while using large topologies, a non-contiguous data structure is used to store the nodes for better scalability. nodes are ketp in random memory location $\operatorname{Loc}_{k}$. The ID is calculated using rules in Eq. 6 and are kept along with the nodes data. A hash table maps index to location, thus the search time is reduced to $O(1)$. Figure 4 depicts the process.

2) Optimising Route-Forest formation: Mroute is a very expensive algorithm in terms of space consumption, while generating a Route-Forest. The algorithm is invoked $O\left(n^{2}\right)$ times. The calculation of Route-tree for any arbitrary pair of nodes is computationally independent, since they share common data-structure $A D J, N C O S T$. This satisfies the criteria to execute them in parallel without any Race-condition (as no write operation on global structures takes place). Therefore, each $T_{i, j} \forall(i, j) \in V^{2}$ is computed parallelly in their individual threads. Also, $T_{i, j}$ can also be realized by reversing $T_{j, i}$ with $O\left(n^{2}\right)$ time.
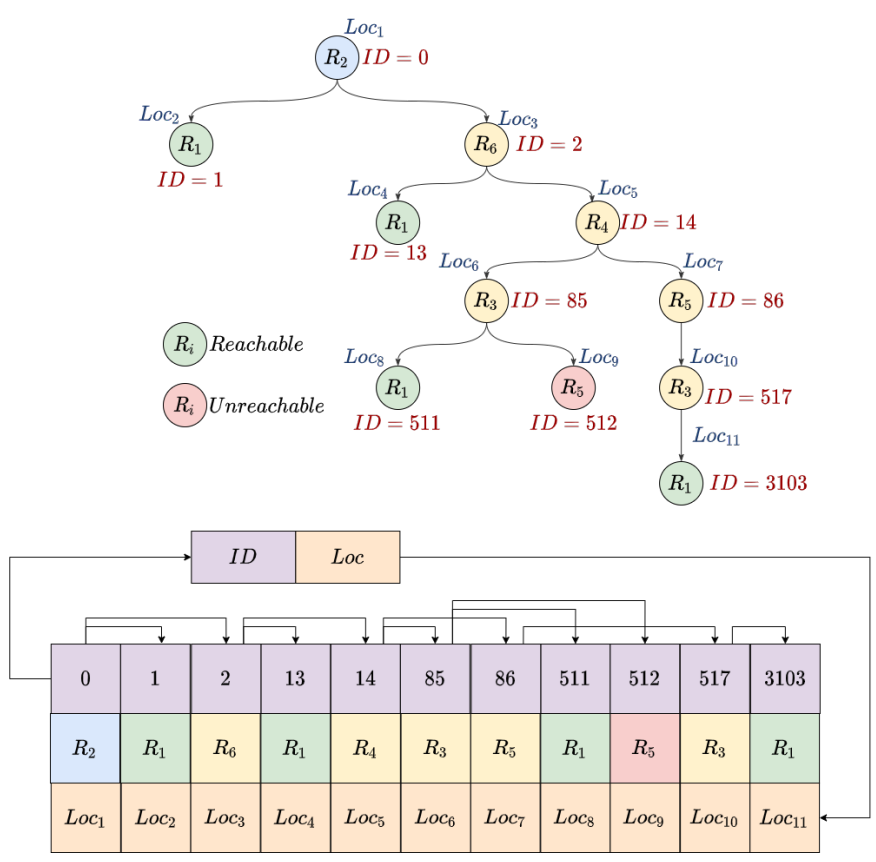

Fig. 4. Dynamic Array-list with hash-table organization for fast searching. $\operatorname{Loc}_{i}$ is the virtual memory location, that holds the router object $R_{j}$ with ID $k$. Hash table maps an ID to its location

\section{Complexity Analysis}

Lemma 1. MRoute is deterministic and loop-free

Proof. The proof is two part, we will first show that the algorithm is loop-free, which will lead us to prove that it is deterministic. Also, properties mentioned in Section III(A.5) is referred in this proof.

MRoute selects Children $C_{k}$ of an non-leaf vertex $v_{k}$ by filtering them with $A D J\left(v_{k}\right)-\operatorname{ANSC}\left(v_{k}\right)$. Therefore, any internal vertex $v_{i}$ if visited by a branch, can't be a part of its descendant. Hence, it satisfies property $4, \operatorname{ANSC}\left(v_{k}\right) \cap$ $\operatorname{DESC}\left(V_{k}\right)=\phi$.

Since, the algorithm is loop free, thus maximum depth the tree can recur is the diameter $(d)$ of $G(V, E)$. Since $1 \leq d \leq|V|$, the recursive process has a deterministic termination.

\section{Lemma 2. MRoute is NP-hard and Traceable}

Proof. We first prove the recurrence relation corresponding to the algorithm falls under the exponential class, then reduce it into the Satisfiability problem to prove it is NP-hard and Traceable.

Let us assume the average branching factor for $T_{s, d}$ be $\bar{b}$ which equals to the mean degree of $G(V, E)$. The algorithm takes $O(1)$ time to fetch $A D J\left(v_{k}\right)$ and $O\left(\log _{b}|V|\right)$ for $A N S\left(V_{k}\right)$. With Memoization, these calls can be made fixed through the run-time. Recursion is then invoked as many as $b$ therefore,

$$
T(n)=\left\{\begin{array}{ll}
0 & \text { if } n=1, \\
1 & \text { if } n=2 \\
b \cdot T(n-1)+\log _{b}|V| & \text { otherwise }
\end{array}\right\}
$$

Using Master theorem [28], it can be shown $T(n)=$ $O\left(b^{n} \log _{b}|V|\right)$. 


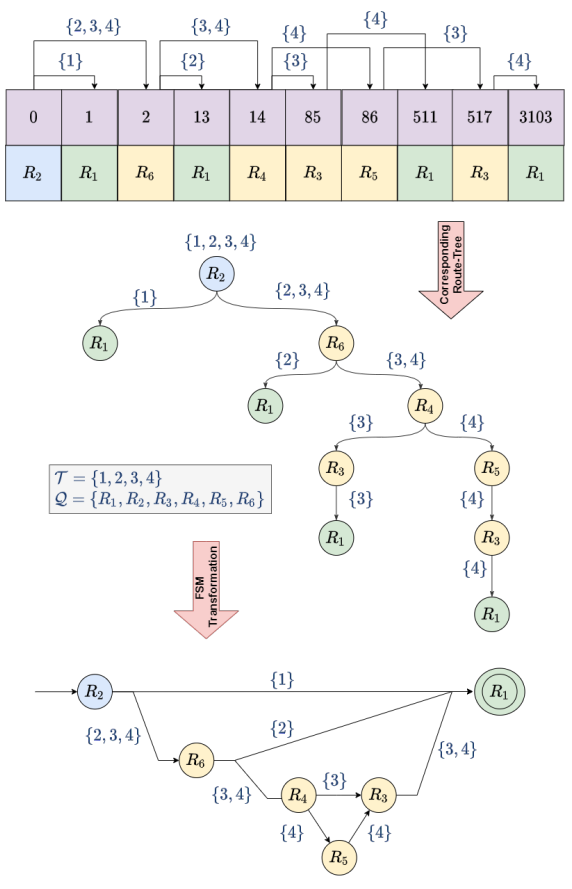

Fig. 5. Implementation of Route-Tag and generating FSM form route tree. The process depicts the transformation of data-structures from the Route-Tree to Route State Graph

To prove the reduction, we'll use an intuitive approach. Since $|E|$ is finite, and $G$ is connected, there exists a path $\operatorname{Path}(i, j)$ between all pair of vertices $v_{i}, v_{j}$. Therefore a path $\operatorname{Path}(i, j)=\{e \in E\} \subseteq 2^{E}$. Every path can be encoded by a binary string of length $|E|$, setting 1 s to all inclusive edges and 0 s to exclusive ones. Hence, it is reduces to an $n-S A T$ problem where $n=|E|$. Thus MRoute is NP-Hard.

Lemma 1 also proves the algorithm is deterministic, hence it is traceable.

1) FSM model and Route-tag: Let $\mathcal{M}\left(\mathcal{Q}, \mathcal{T}, \delta, q_{0}, \mathcal{F}\right)$ be a deterministic finite state machine such that. $Q$ is a Finite, non-empty set of states $(Q=\mathcal{V}), \mathcal{T}$ is a Finite, non-empty set of Route-Tags $Q \cap \mathcal{T}=\phi, \delta$ is the Transition function $\delta: Q \times \mathcal{T} \rightarrow Q, q_{0}$ is the Initial State, $q_{0}=v_{s} \in Q$ and $\mathcal{F}$ is a Finite, non-empty set of Final state(s), $\mathcal{F}=\left\{v_{d}\right\} \subseteq Q$

Any RouteTree tree has unique paths between root and leaves. An identifier called Route-Tag tags each path. This compresses the exponentially large Route-Tree into state machine of size $O(|V|)$, We term this transformation $\mathcal{F}: T_{i, j} \rightarrow$ $\mathcal{M}_{i, j}$ Route State Transformation Function (RSTF) and $\mathcal{M}_{i, j}$ as Route State Graph (RSG). Figure 5 depicts the transformation with changes in the data-structures.

\section{E. Path Matrix}

A path-matrix $\mathcal{P}=V^{2}$ is defined as, $\left\{p_{i, j} \in \mathcal{P}\right\}=\mathcal{M}_{i, j}$. Every valid traversal in $\mathcal{M}_{s, d}$ corresponds to a feasible route between $R_{s}, R_{d}$. We propose two methods to encode the RSG.

1) Encode as Grammar: In this approach, the state machine is encoded into a set of production rules called Grammar
$\mathcal{G}(\mathcal{V}, \mathcal{T}, \mathcal{P}, s)$. This mode of encoding is useful when the routes are generated either as patterns or Regular Expressions. A grammar $\mathcal{G}$ is expressed as a quadruple where, $\mathcal{V}$ Set of Non-terminals $=Q \subseteq V, \mathcal{T}$ set of terminals (Route-tags), $\mathcal{P}$ set of Regular production rules, $s$ start symbol.Encoding RSG into its grammar, summarizes the routes and parsing-ability is enforced using regular expressions.

2) Encode as Tag-Cost Table: The Tag-Cost-table TCT = $\mathcal{T} \times E$ is a binary matrix, each row identifies one route-tag $\left(t_{k} \in \mathcal{T}\right)$ and it's corresponding edge set. The column-sum tells how many routes-tags are sharing a given edge (typically used for load-balancing). The Tag-Cost function is formulated in Equation 8 and the Tag-Cost table in Table II. A Min-heap implementation of storing the tag-costs takes $O(1)$ time to return the best route and $O\left(\log _{\bar{b}}|V|\right)$ time to reorder them.

$$
c_{s, d}^{\left(t_{k} \in \mathcal{T}\right)}(t)=\sum_{(i, j) \in E}\left(c_{i, j}(t) \times T C T\left[t_{k}\right]\right)
$$

Encoding RSG into TCT does leverage the reactive routeresponse mechanism, due to its constant time search for best route. Also, the tabular structure makes it easy to program and alter with varying edge costs.

\begin{tabular}{|l|l|l|l|l|l|l|l|l|l|}
\hline Tags & $e_{1,2}$ & $e_{1,3}$ & $e_{1,6}$ & $e_{2,6}$ & $e_{3,4}$ & $e_{3,5}$ & $e_{4,5}$ & $e_{4,6}$ & Cost \\
\hline $\mathbf{1}$ & 1 & 0 & 0 & 0 & 0 & 0 & 0 & 0 & $c_{1,2}^{(1)}(t)$ \\
\hline $\mathbf{2}$ & 0 & 0 & 1 & 1 & 0 & 0 & 0 & 0 & $c_{1,2}^{(2)}(t)$ \\
\hline $\mathbf{3}$ & 0 & 1 & 0 & 1 & 1 & 0 & 0 & 1 & $c_{1,2}^{(3)}(t)$ \\
\hline $\mathbf{4}$ & 0 & 1 & 0 & 1 & 1 & 1 & 1 & 0 & $c_{1,2}^{(3)}(t)$ \\
\hline Share & 1 & 2 & 1 & 3 & 2 & 1 & 1 & 1 & \\
\multicolumn{10}{c}{ TABLE II } \\
\hline
\end{tabular}

\section{ESTIMATION OF RELIABILITY USING RNN}

As the normalised costs matrix (NCOST) varies over time (due to the variation of node or link cost), it creates a time series matrix. However, the matrix comprises individual normalised links which vary independently and does not provide performance analytics directly. Therefore, first we segregate each link and treat them as individual time series. Then, unlike predicting the traffic pattern or load, we focus more on predicting the trend. One of the challenges regards the online training in dynamic environment, A trained neural network often rejects to adapt sudden changes as outlier. Therefore, we aim to model the network dynamics by the degree of volatility of individual links.

\section{A. Sharpe-Ratio based approximation}

In fanance, the Sharpe-Ratio [29] is a widely used metrics in portfolio management that measures the volatility of a stock and estimates the risk associated with it. It is defined as the ratio of the Sample-Mean and the Sample-Standard-deviation of a set and is proportional to the volatility. The approximation steps are as follows, 


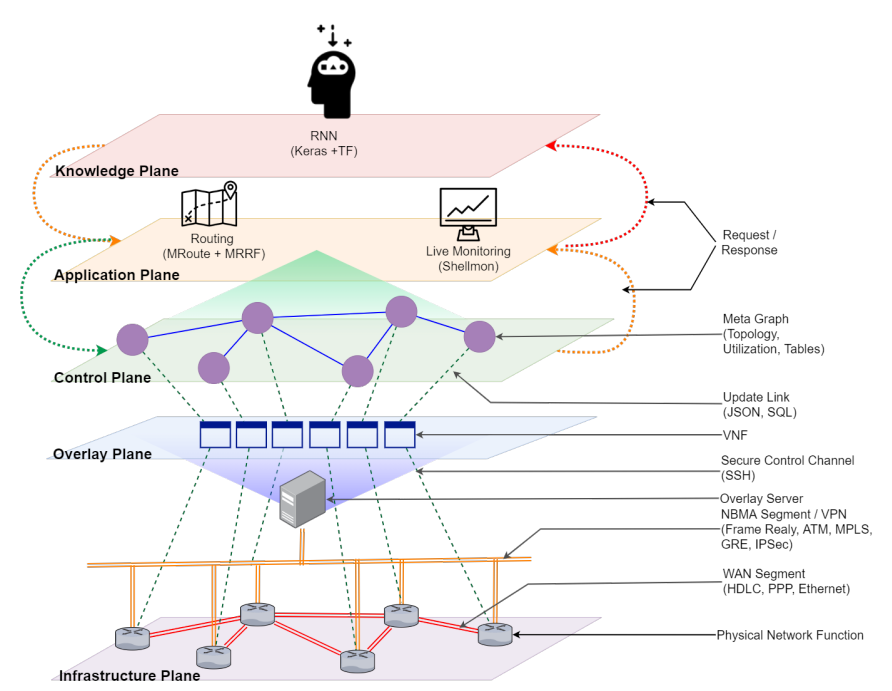

Fig. 6. Deployment diagram of the Test-bed. Infrastructure plane holds routers, overlay server receives monitoring information and spawns VNFs per Router. Control plane discover topology and application plane operates on it. Knowledge plane is for self-learning however beyond the scope of the context.

1) Calculate Volatility $V_{i, j}(t)$ of each $C_{i, j}(t)$ with a user defined window size $W$ rolling over time $t$.

$$
\mathcal{V}_{i, j}(t)=\frac{\overline{C_{i, j}^{\prime}([t-w: t])}}{S D\left(C_{i, j}([t-w: t])\right)} \forall(i, j) \in E
$$

2) Estimate the edge-wise hypothesis functions $\mathbf{h}_{\mathbf{i}, \mathbf{j}} \in \mathcal{H}$ as a auto-regressive function using an RNN with a period of $W$.

$$
\mathcal{V}_{i, j}(t+1)=\mathbf{h}_{\mathbf{i}, \mathbf{j}}\left(\mathcal{V}_{i, j}[t-w: t]\right)
$$

3) Use $V_{i, j}(t+1)$ as a metric to choose the best path. The proposed model uses offline training to build the initial model and thereafter uses online training to update it. We define a cutoff value $\epsilon>30 \%$.

\section{IMPLEMENTATION}

Figure 6 depicts the deployment-diagram of our test-bed. A multi-tier approach is conceived for operational and functional segregation. The SDN philosophy of decoupling control and data plane has been the core design principal for the proposed architecture. However, the Knowledge plane has been integrated on top to support SON capabilities.

\section{A. A 5-Tier KDN Test-Bed}

The architecture supports network-automation and SON. It finds optimal route using MRoute (Self-Optimization), then installs them to the underlying nodes by pushing device-specific configuration into the edge-devices (SelfConfiguration) and guarantees a most-reliable route by keep updating them over the time (Self-Healing). Thus it meets all the three criteria of SON. The following explains the working of the layers. Please refer to the implementation details including connection API and algorithm's code for more details [30].
1) Infrastructure Plane: This layer hosts physical and/or emulated network nodes (e.g. routers, L2/L3 switches etc.). Routers are connected to Overlay-plane securely using IPSec-DMVPN tunnels to exchanges any control-traffic.

2) Overlay Plane: This layer interfaces between the infrastructure and control plane. For each downstream router, a VNF process (agent) maintains a secure link (using SSH) to monitor the resource utilization. Additionally, it also injects configuration commands. We use Napalm, library to automate the routes.

3) Control Plane: Resource and topology information are fused to generate the meta-graph in the control plane. RESTConf is used to interface with overlay plane below and application plane above.

4) Application Plane: Application plane brings modularity in the architecture, such as Monitoring, Routing, etc. MRoute is one of such application. However, there are other functions such as migration and monitoring which are beyond the scope of the context of this paper.

5) Knowledge Plane: The knowledge plane leverages the KDN paradigm. This component takes care of all data preprocessing, offline and online training. It returns a trained model initially as an outcome of offline training. However, the model gets updates during online training whenever the trend changes. KDN functionalists can be divided into four main unit.

- Pre-processing: It performs data acquisition, data quality checks and validations, imputing and standardization. Typically $70 \%$ of the overall process time is spent on this phase.

- Offline Training: The offline training starts by dividing the data into training, validation and testing for the machine learning model. It utilizes the historical data from the repository to train the model, predicts the networking characteristics to produce a decision.

- Online Training: It is used when the data is generated in a form of a sequence (such as time series). Network resource utilization is a from of a time series (NCOST) of a $n \times n \times t$ tensor. where $n$ be the number of nodes nodes and $t$ be the time.

- Modelling: The learning algorithm learns from the fed data-set, and generates a model for prediction. Since the problem can be classified as a time series prediction type, RNN is chosen as the base architecture.

\section{B. Performance analysis of MRoute}

The comparative analysis between MRoute, DUAL and SPF (Figure 7 ) benchmarks algorithms using six parameters as discussed in Section 6.2., In this section we present a comprehensive explanation to the results. Subplot 7(A) compares the time complexity with respect to the size of the network, outcomes are plotted in log-scale therefore MRoute shows an exponential growth, as shown in Lemma 2; in comparison, 

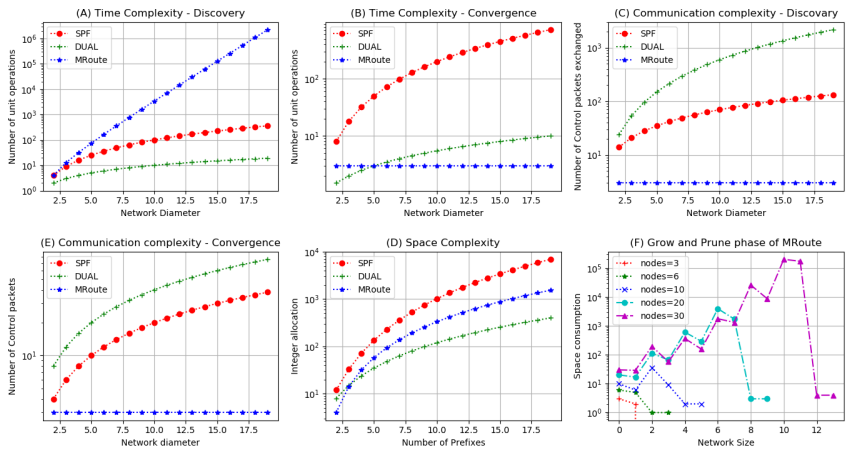

Fig. 7. Experimental Results and Comparison MRoute against SPF and DUAL using the following parameters (A)Time Consumption to computing paths, (B) Time consumption to converge, (C) Control traffic for topology synchronization, (D) Space consumption for topology maintenance (E) Control traffic for convergence, (F) Route-Tree size .

DUAL and SPF which are bounded above by $O\left(n^{2}\right)$. Due to the diffusion-computation model and the presence of feasiblesuccessor, DUAL goes less deep into the convergence state than of SPF. We tuned the SPF to run on each down-stream topology in parallel, simulating a multi-area OSPF network. It seems initially that DUAL is the optimum than its competitors. MRoute calculates all possible paths in advanced. Therefore, in the long run if the topology remains unaltered, it would never enter a re-convergence process, which is not the case of the rest two. This situation is shown in the subplot 7(B), where the random link failure scenario (Section 6.1) causes SPF to re-converge every time, DUAL shows a better result as in some of the cases feasible-successor exists or a neighbour replies with route much before the query reaches the network boundary. However, MRoute shows a constant reading here as it is an $O(1)$ task that require a fixed number of operation involving querying and getting reply for the next best route. The process can be thought as a generalised case of DUAL where all backup routes are ranked and listed.

The communication complexity measures the number of packets exchanged between the nodes while discovering or converging into the network. In case of SPF and DUAL, the algorithms are inherently distributed, therefore the local routes are advertised, queried during re-convergence and polled for their liveliness using reliable updates and Hello protocols respectively. Since OSPF uses link-state model, the total number of packets exchanged is higher than that of Distance-vector based on EIGRP. MRoute is designed as a centralised routing algorithm. Therefore it does not exchange any discovery or update messages with other nodes. It updates only the controller which is logically one hop away, This justifies the subplot $7(\mathrm{C}, \mathrm{E})$.

The state-model representation of the route-forest reduces the space consumption of MRoute drastically by tagging routes as a fixed length binary vector of edges with RouteID. However, while generating the Route-Tree, it consumes memory in an exponential rate. After the complete forest is generated, the state model gets built which compresses them into tables and relinquishes the memory (subplot $7(\mathrm{~F})$ ). Space complexity of MRoute sits between SPF and DUAL as OSPF maintains

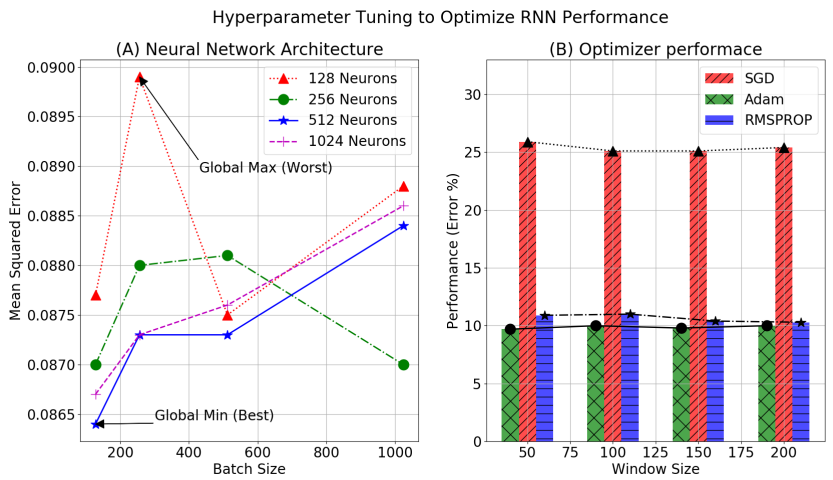

Fig. 8. (A) Comparison of accuracy (by mean squared error) with four network setups $(128,256,512 \& 1024)$, the Global optima is reached with 128 Neuron at a batch size of 512. (B) compares three optimizer algorithms (SGD, Adam \& RMSPROP), over a varying window size of [20 - 200], on which Adam gives best result on average

identical link-state database for all nodes and EIGRP topology tables lists the successor and feasible-successors for each destination prefix depicted in subplot $7(\mathrm{~F})$.

\section{RNN Architecture}

In this section, the design of the machine learning architecture is presented. We also introduce a few techniques used like hyper-parameters fine-tuning and choosing the best optimization algorithm.

1) Hyper-Parameter Tuning: In this phase, the Hyperparameters such as Batch-size and number of neuron are tuned from experimental data. Figure 8(A) depicts testing Mean Squared Error (MSE) cross-validation for 3 layers on a Deep RNN using 200 epochs. The reason for this was to choose the appropriate number of neurons and the batch size for the training and validation data-sets, error rate is measured using Mean Squared Error (MSE). As highlighted in bold, the optimum hyper-parameters have been 128 neurons and 512 batch size at $0.08 \mathrm{MSE}$.

2) Optimization Algorithm: Figure 8(B) shows a comparison of the various optimizers proposed by [31] . For the LSTM model, different sets of window sizes are tested. Three main variants Gradient Descent (SGD, ADAM \& RMSPROP) are compared. As a proof of concept, results shoe that predicting with a $200 \mathrm{~ms}$ window size using Adam can achieve a mean error rate of $10 \%$.

3) Scoring: The proposed technique performs traffic prediction on the normalized reliability of the links. The result shows that, with the appropriate hyper-parameters, reliability can be estimated with a mean $90 \%$ accuracy.

\section{Online Learning}

The online learning phase receives constant feedback from the network. If the predicted reliability deviates from the actual one, within a given threshold, the RNN needs to relearn to adjust its weights. The re-learning process takes place for multiple edges simultaneously. Hence, the tuning needs to be optimised. We use TensorFlow's Early-Stopping 

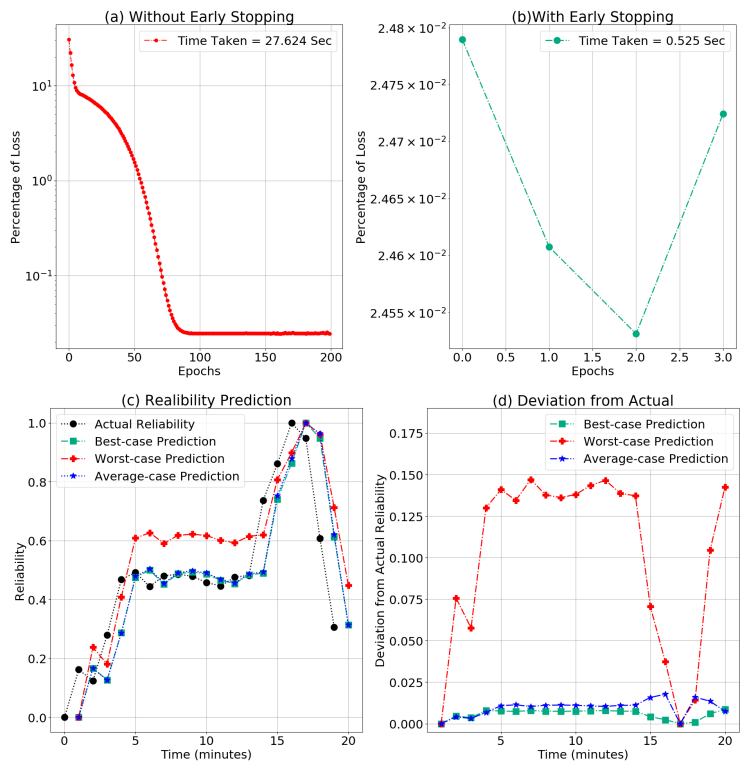

Fig. 9. Evaluation of the Online-Learning, (a) Learning time with 200 epochs (b) Accelerated learning with Early-Stopping enabled (c) Comparing timeseries prediction of reliability in Best, Average and Worst case scenario (d) compares the deviation in log-scale, also shows the comparison is distinctive when there is less fluctuation

feature to accelerate the learning process, by monitoring the loss function's value and breaking the iteration whenever the loss converges to a value. Therefore, the learning process doesn't need to run for all the epochs. Figure 9(a) shows the loss function's characteristics spanning for 200 epochs which took 27.6 Seconds to complete learning. However it can be noticed that the function actually settles around 55'th epoch and stays constant since then. The acceleration is depicted in Figure9(b) where using early stopping the same network could be retrained in just 0.53 seconds. Thus it reduces exponentially the time consumption of re-training the RNN, making it feasible for online training.

A more comprehensive comparison between the actual and predicted reliability are shown in Figure 9(c,d). The first compares the best, worst and average cases, sampling them down to a set of 20 instances, collected over period of 20 minutes of online learning. The results show discrimination is prominent when there is less fluctuation in the data sets, it's more comprehensive when the deviation is plotted in log-scale (Figure 9(d))

\section{E. Rapid Convergence and Co-relation to Sharpe Ratio}

Figure 10 depicts the varying reliability of five edge nodes over a time period of 350 stamps each of 10 seconds. The $\log$ scale is used to magnify the variation. Over the time, there are three nodes that have come up as the most reliable in the order of $\mathrm{None}_{2}, \mathrm{Node}_{4}, \mathrm{Node}_{5}$ and again Node 2 . During the experiment, we have emulated this dynamics by randomly altering various node and edge attributes. This causes the network to be extremely chaotic and the routing protocols to re-converge frequently. An effect that appears clearly in Figure7(A,E). MRoute has shown an $O(1)$ time

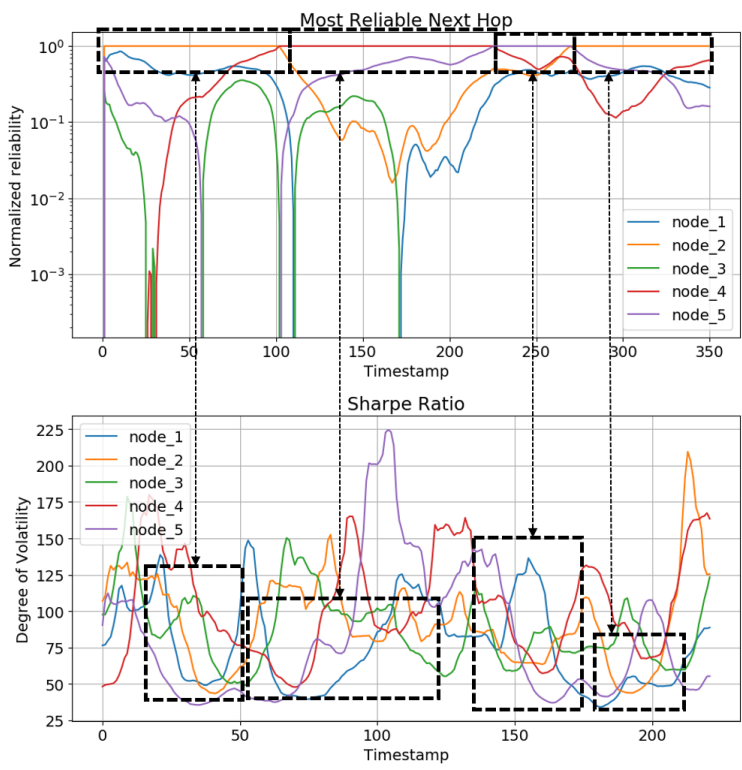

Fig. 10. Demonstration of Self-Healing through rapid-convergence: At timestamp [0-100] $\mathrm{Node}_{2}$ is most reliable as the corresponding rolling SharpeRatio has maximum descending gradient calculated on 100 timestamps. Similar pattern can be noticed for $\mathrm{Node}_{4}$ during [100-240], Node 5 during [240-270] and $\mathrm{Node}_{2}$ during [270-350]. The correlation is analytical however the RNN learns it.

convergence as routes are not only chosen in constant time. Additionally, the most-reliable node is switched instantly. A clear correlation between the learnt reliability and the sharperatio is also drawn using the relative dotted-boxes. As the sharpe-ratio measures the degree of volatility, every time it meets a rapid depression. The corresponding router is chooses as most reliable. During the training, the RNN captures this trend and predicts accordingly. We set the window size of 100 time-stamps thus a offset of 100 can be seen in the time-axis of the two plots.

\section{CONCLUSION}

In this paper we propose a cognitive routing framework for KDN to support IoT applications for Industry 5.0. The framework uses an Shortest Path Algorithm names MRoute, that proactively computes all-possible paths between all pairs of nodes. Further, it uses Sharpe-Ratio to measure volatility of each link and RNN with LSTM to learn trend. The framework uses online learning to tackle any dynamic network behaviour. Result shows that the MRoute gives a constant-time convergence.

\section{ACKNOWLEDGMENT}

The work is undertaken in the context of the "SelfOrganization Toward Reduced Cost and Energy Per Bit for Future Emerging Radio Technologies" with contract number 734545.The project has received research funding from the H2020-MSCA-RISE-2016 European Framework Program. 


\section{REFERENCES}

[1] K. Nisar, E. R. Jimson, M. H. A. Hijazi, I. Welch, R. Hassan, A. H. M. Aman, A. H. Sodhro, S. Pirbhulal, and S. Khan, "A survey on the architecture, application, and security of software defined networking: Challenges and open issues," Internet of Things, vol. 12, p. 100289, 2020. [Online]. Available: https://www.sciencedirect.com/science/article/pii/S2542660520301219

[2] P. V. Klaine, M. A. Imran, O. Onireti, and R. D. Souza, "A survey of machine learning techniques applied to self-organizing cellular networks," IEEE Communications Surveys Tutorials, vol. 19, no. 4, pp. 2392-2431, 2017.

[3] D. D. Clark, C. Partridge, J. C. Ramming, and J. T. Wroclawski, "A knowledge plane for the internet," in Proceedings of the 2003 Conference on Applications, Technologies, Architectures, and Protocols for Computer Communications, ser. SIGCOMM '03. New York, NY, USA: Association for Computing Machinery, 2003, p. 3-10. [Online]. Available: https://doi.org/10.1145/863955.863957

[4] R. Hajlaoui, H. Guyennet, and T. Moulahi, "A survey on heuristic-based routing methods in vehicular ad-hoc network: Technical challenges and future trends," IEEE Sensors Journal, vol. 16, no. 17, pp. 6782-6792, 2016.

[5] O. G. Aliu, A. Imran, M. A. Imran, and B. Evans, "A survey of self organisation in future cellular networks," IEEE Communications Surveys \& Tutorials, vol. 15, no. 1, pp. 336-361, 2012.

[6] G. P. Fettweis, "A 5g wireless communications vision," Microwave Journal, vol. 55, no. 12, pp. 24-36, 2012.

[7] J. G. Andrews, S. Buzzi, W. Choi, S. V. Hanly, A. Lozano, A. C. Soong, and J. C. Zhang, "What will 5g be?" IEEE Journal on selected areas in communications, vol. 32, no. 6, pp. 1065-1082, 2014.

[8] P. Wainio and K. Seppänen, "Self-optimizing last-mile backhaul network for 5g small cells," in 2016 IEEE International Conference on Communications Workshops (ICC). IEEE, 2016, pp. 232-239.

[9] P. V. Klaine, M. A. Imran, O. Onireti, and R. D. Souza, "A survey of machine learning techniques applied to self-organizing cellular networks," IEEE Communications Surveys \& Tutorials, vol. 19, no. 4, pp. 2392-2431, 2017.

[10] W. T. Zaumen and J. J. Garcia-Luna-Aceves, "Loop-free multipath routing using generalized diffusing computations," in Proceedings. IEEE INFOCOM '98, the Conference on Computer Communications. Seventeenth Annual Joint Conference of the IEEE Computer and Communications Societies. Gateway to the 21st Century (Cat. No.98, vol. 3, 1998, pp. 1408-1417 vol.3.

[11] E. W. Dijkstra, "A note on two problems in connexion with graphs," Numerische Mathematik, vol. 1, no. 1, pp. 269-271, Dec 1959. [Online]. Available: https://doi.org/10.1007/BF01386390

[12] J. Strassner, M. O'Foghlu, W. Donnelly, and N. Agoulmine, "Beyond the knowledge plane: An inference plane to support the next generation internet," in 2007 First International Global Information Infrastructure Symposium, 2007, pp. 112-119.

[13] J. Xie, F. R. Yu, T. Huang, R. Xie, J. Liu, C. Wang, and Y. Liu, "A survey of machine learning techniques applied to software defined networking (sdn): Research issues and challenges," IEEE Communications Surveys Tutorials, vol. 21, no. 1, pp. 393-430, 2019.

[14] Z. M. Fadlullah, F. Tang, B. Mao, N. Kato, O. Akashi, T. Inoue, and K. Mizutani, "State-of-the-art deep learning: Evolving machine intelligence toward tomorrow's intelligent network traffic control systems," IEEE Communications Surveys Tutorials, vol. 19, no. 4, pp. 2432-2455, 2017.

[15] M. Chen, U. Challita, W. Saad, C. Yin, and M. Debbah, "Artificial neural networks-based machine learning for wireless networks: A tutorial," IEEE Communications Surveys Tutorials, vol. 21, no. 4, pp. 3039-3071, 2019.

[16] M. R. Haque, S. C. Tan, Z. Yusoff, C. K. Lee, and R. Kaspin, "Ddos attack monitoring using smart controller placement in software defined networking architecture," in Computational Science and Technology, R. Alfred, Y. Lim, A. A. A. Ibrahim, and P. Anthony, Eds. Singapore: Springer Singapore, 2019, pp. 195-203.

[17] Y. Zhao, Y. Li, X. Zhang, G. Geng, W. Zhang, and Y. Sun, "A survey of networking applications applying the software defined networking concept based on machine learning," IEEE Access, vol. 7, pp. 95 397$95417,2019$.

[18] L. Yanjun, L. Xiaobo, and Y. Osamu, "Traffic engineering framework with machine learning based meta-layer in software-defined networks," in 2014 4th IEEE International Conference on Network Infrastructure and Digital Content, 2014, pp. 121-125.
[19] A. Azzouni, R. Boutaba, and G. Pujolle, "Neuroute: Predictive dynamic routing for software-defined networks," in 2017 13th International Conference on Network and Service Management (CNSM), 2017, pp. $1-6$.

[20] Á. López-Raventós, F. Wilhelmi, S. Barrachina-Muñoz, and B. Bellalta, "Machine learning and software defined networks for high-density wlans," 2018.

[21] S. Sendra, A. Rego, J. Lloret, J. M. Jimenez, and O. Romero, "Including artificial intelligence in a routing protocol using software defined networks," in 2017 IEEE International Conference on Communications Workshops (ICC Workshops), 2017, pp. 670-674.

[22] F. Francois and E. Gelenbe, "Optimizing secure sdn-enabled interdata centre overlay networks through cognitive routing," in 2016 IEEE 24th International Symposium on Modeling, Analysis and Simulation of Computer and Telecommunication Systems (MASCOTS), 2016, pp. 283-288.

[23] S. Lin, I. F. Akyildiz, P. Wang, and M. Luo, "Qos-aware adaptive routing in multi-layer hierarchical software defined networks: A reinforcement learning approach," in 2016 IEEE International Conference on Services Computing (SCC), 2016, pp. 25-33.

[24] ONF, "OpenFlow Switch Specification," pp. 11-200, mar 2015. [Online]. Available: https://www.opennetworking.org/wpcontent/uploads/2014/10/openflow-switch-v1.5.1.pdf

[25] "ONLP APIs for Applications OpenNetworkLinux." [Online]. Available:
http://opencomputeproject.github.io/OpenNetworkLinux/onlp/applications

[26] R. Moskowitz, D. Karrenberg, Y. Rekhter, E. Lear, and G. J. de Groot, "Address Allocation for Private Internets," RFC 1918, Feb. 1996. [Online]. Available: https://rfc-editor.org/rfc/rfc1918.txt

[27] S. Ghosh, T. Dagiuklas, and M. Iqbal, "Energy-aware ip routing over sdn," in 2018 IEEE Global Communications Conference (GLOBECOM), 2018, pp. 1-7.

[28] C. Yap, "A real elementary approach to the master recurrence and generalizations," in TAMC, 2011.

[29] W. F. Sharpe, "The Sharpe Ratio," The Journal of Portfolio Management, vol. 21, no. 1, pp. 49-58, 1994. [Online]. Available: https://web.stanford.edu/ wfsharpe/art/sr/sr.htm

[30] S. Ghosh, "GitHub - rishiCSE17/SO-KDN: Self Organised Knowledge Defined Netwrok." [Online]. Available: https://github.com/rishiCSE17/SO-KDN

[31] M. Wang, Y. Cui, X. Wang, S. Xiao, and J. Jiang, "Machine learning for networking: Workflow, advances and opportunities," IEEE Network, vol. 32, no. 2, pp. 92-99, mar 2018. 


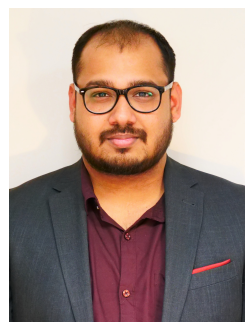

Mr. Saptarshi Ghosh received his B.Sc.(Hons.) in Computer Science and M.E. in Software Engineering from University of Calcutta and Jadavpur University, India, respectively followed by an M.Sc. in Smart Networks from the University of the West of Scotland, U.K. He is currently pursuing the $\mathrm{Ph} . \mathrm{D}$. degree in computer science and informatics at London South Bank University and working as a module leader several core CS modules at the same department. He has been a recipient of GATE and Erasmus-Mundus Scholarship. His Ph.D. research is under the EU-Horizon 2020 project, supported by Marie-Curie Fund with the research area focused in machine learning's application to self-organized SDN for 5G and Beyond. Saptarshi is a JNCIA (DevOps) certified and has worked as a Software Developer and Network Engineer. He has contributed into several research and software engineering project funded by Erasmus+, Innovate UK and Defence Science \& Technology Laboratory. His research interests include SD-WAN, network programmability and automation, cognitiverouting and deep reinforcement learning.

Prof. Tasos Dagiuklas is a leading researcher and expert in the fields of smart Internet technologies. $\mathrm{He}$ is the leader of the Smart Internet Technologies (SuITE) research group at the London South Bank University where he also acts as the Head of $\mathrm{Cog}_{-}$ nitive Systems Reseach Centre. Tasos received the Engineering Degree from the University of PatrasGreece in 1989, the M.Sc. from the University of Manchester- UK in 1991 and the Ph.D. from the University of Essex-UK in 1995, all in Electrical Engineering. He has been a principal investigator, co-investigator, project and technical manager, coordinator and focal person of more than 20 internationally $R \& D$ and Capacity training projects in the areas of Fixed- Mobile Convergence, 4G/5G networking technologies, VoIP and multimedia networking. His research interests lie in the field of Systems Beyond 5G/6G networking technologies, programmable networks, UAVs, V2X communications and cyber security for smart Internet systems.

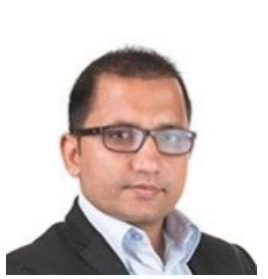

Dr. Muddesar Iqbal completed his PhD Funded by EPSRC Doctoral Training Award for 4G-based Reconfigurable Mobile Healthcare System from Kingston University, the UK in 2010. He has worked with several universities in UK, EU and South East Asia and currently working as a Senior Lecturer with London South Bank University. He has been a principal investigator, co-investigator, project manager, coordinator and focal person of more than 15 internationally teamed research and development, capacity building and training projects, resulting in several patented inventions and commercial products. His areas of research are Internet of Sense for Industry 5.0, Intelligent Autonomous Machines/Robotics, Conversational AI and Chabot's, Personalization/Recommendation, 6G Context-Aware Systems, and Collaborative Cognitive-Communication Systems.

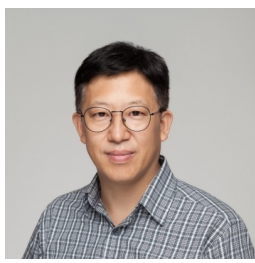

Prof. Xinheng Wang is currently the founding Head of Department of Mechatronics and Robotics. He is an IET Fellow and a senior member of IEEE. $\mathrm{He}$ is also a team leader of Jiangsu Innovation and Entrepreneur Programme. He has broad academic working experience in China, England, Wales, and Scotland for more than 20 years. He has extensive research experience in Internet of Things (IoT), wireless mesh networks, indoor positioning, big data analytics, and applications for smart cities. Along with nearly 30 research projects sponsored from EU, UK EPSRC, Innovate UK, China NSFC, and industry, his research in each area has led to an impactful industrial product. His collaborative research in acoustic localisation with Zhejiang University has won the first place in Microsoft Indoor Localisation Competition in sound group. His current research interests include 6G networks and Industrial Internet of Things, indoor positioning and data services, acoustic localisation, communications and sensing, and smart services for group travellers. 